\title{
Optical solitons in semiconductor quantum dot waveguides
}

\author{
G.T. Adamashvili ${ }^{1,2, a}$, A. Knorr² ${ }^{2}$ and C. Weber ${ }^{3, b}$ \\ 1 Max-Planck-Institut für Physik komplexer Systeme, Nöthnitzer Str. 38, 01187 Dresden, Germany \\ 2 Institut für Theoretische Physik, Nichtlineare Optik und Quantenelektronik, Technische Universität Berlin, \\ Hardenbergstr. 36, 10623 Berlin, Germany \\ 3 Mathematical Physics, Lund University, Box 118, 22100 Lund, Sweden
}

Received 27 June 2007 / Received in final form 15 January 2008

Published online 12 March 2008 - (C) EDP Sciences, Società Italiana di Fisica, Springer-Verlag 2008

\begin{abstract}
A theory of self-induced transparency for a TM-mode propagating in a planar semiconductor waveguide sandwiched between two dielectric media is developed. A transition layer between the waveguide and one of the connected media is described using a model of a two-dimensional sheet of quantum dots. Explicit analytical expressions for the optical soliton in the presence of single-excitonic and biexcitonic transitions are obtained with realistic parameters which can be reached in current experiments.
\end{abstract}

PACS. 42.65.-k Nonlinear optics - 42.65.Tg Optical solitons; nonlinear guided waves - 78.67.Hc Quantum dots

Semiconductor quantum dots (SQDs) as model systems for the study of light-matter interaction have been investigated extensively in the past years. Due to their zerodimensional properties such as discrete energy spectra resulting from the confinement of the charge carriers in all three spatial dimensions, these atomlike structures have been proposed for the use as qubits in quantum information processing [1] as well as for laser devices [2]. In addition, due to their large dipole moments reaching values on the order of $10^{-17}$ esu cm [3], the interaction between SQDs and optical light fields is strongly enhanced in comparison with atomic systems, making them good candidates to study nonlinear optical propagation effects. Nonlinear optical experiments can be performed with pulses of a few hundreds of femtoseconds which are long compared to the inhomogeneous broadening but short enough so that the electron-phonon interaction which acts on a picosecond timescale can be neglected [4-7], offering time scales necessary for coherent interaction. Finally, great progress has been made in the design and control of SQDs for technical devices.

Compared to atomic systems, typically described by noninteracting two-level systems, SQDs experience manybody effects (such as exciton-exciton interactions leading to the formation of biexcitonic states) which are unknown in atomic systems. In addition, the observation of optical coherence effects in ensembles of SQDs is influenced by the inhomogeneous line broadening due to dot size fluc-

\footnotetext{
a Permanent address: Georgian Technical University, Kostava St. 77, 0177 Tbilisi, Georgia

b e-mail: carsten.weber@teorfys.lu.se
}

tuations, leading to an inhomogeneous single-exciton and biexciton level broadening, with a full width at half maximum of typically more than several tens of meV [8]. Thus, nonlinear optical effects such as SIT differ substantially in SQDs.

In atomic systems, resonant solitons can be formed within the nonlinear McCall-Hahn mechanism [9] if the conditions for self-induced transparency (SIT) are fulfilled: $\omega T \gg 1, T \ll T_{1,2}$, where $T$ and $\omega$ are the width and frequency of the pulse and $T_{1}$ and $T_{2}$ are the longitudinal and transverse relaxation times of the atoms, respectively. When the area of the pulse $\Theta>\pi$, a soliton ( $2 \pi$ pulse) is generated. It was shown in reference [10] that solitons can form in waveguides covered with thin films of homogeneously broadened two-level atoms at exact resonance.

In semiconductors, experimental observations of selfinduced transmission on a free exciton resonance in CdSe [11] and in InGaAs quantum dot waveguides [8] have been reported. Theoretically, the effect of SIT for solitons in an inhomogeneously broadened SQD ensemble in the presence of single-excitonic and biexcitonic transitions has been investigated numerically in reference [12]. The results have demonstrated that intense optical pulses have properties similar to McCall-Hahn's $2 \pi$ pulse and can propagate in realistic dot samples without suffering strong losses. Rabi oscillations in quantum dots as a prerequisite for resonance solitons [6] as well as nonlinear pulse propagation effects in quantum wells [13] have also been discussed numerically.

Analytical expressions for the parameters of resonance breathers, which form for pulse areas $\Theta \ll 1$, for plane 
waves in SQDs have been presented in reference [14]. In reference [15], both the properties of SIT solitons for plane waves in inhomogeneously broadened SQDs as well as the propagation of optical breathers in a waveguide containing SQDs have been considered. The properties of nonlinear surface waves at the boundary between two connected media with permittivities of opposite signs have been investigated in $\left(A^{I I I} B^{V}\right)$ semiconductors in reference [16]. There, SIT for surface optical waves has been considered under the condition that a small concentration of SQDs forms a thin transition layer between the connected media, and explicit analytical expressions for the parameters of resonance optical surface solitons have been presented.

The purpose of the present article is to theoretically investigate the processes of formation of optical solitons under the condition of SIT in a semiconductor waveguide. Similar aspects of soliton-like pulses have been investigated experimentally [8], but corresponding theoretical investigations have not been carried out up to now. Thus, from an experimental point of view, this situation is more interesting than the corresponding bulk soliton solution [15]. Compared to the existence of stable breather solutions in semiconductor waveguides, where the SQDs are distributed homogeneously in the central medium, here we consider a two-dimensional sheet of inhomogeneously broadened SQDs which forms a transition layer on one of the surfaces of the planar waveguide. In comparison to the small-amplitude breather of reference [15] which is a solution of the nonlinear Schrödinger equation under linear boundary conditions, the waveguide soliton satisfies the Maxwell-Bloch equations with nonlinear boundary conditions.

We consider a TM-mode in the case when an optical pulse with width $T$ and frequency $\omega \gg T^{-1}$ is propagating along the positive $z$ axis. The planar waveguide of thickness $h$ (medium 2) with permittivity $\varepsilon_{2}(\omega)$ is sandwiched between two isotropic media $(x<0$ : medium 1 and $x>h$ : medium 3$)$ with permittivities $\varepsilon_{1}(\omega)$ and $\varepsilon_{3}(\omega)$, respectively. A thin transition layer of thickness $d \ll \lambda$ containing a small concentration of SQDs forms the boundary between media 2 and 3 , where $\lambda$ is the length of the optical waveguide mode.

For a waveguide TM-mode, the strength of the electric field $\boldsymbol{E}=\left(E_{x}, 0, E_{z}\right)$ lies in the $x-z$ plane perpendicular to the boundary of division between the connected media. The strength of the magnetic field $\boldsymbol{H}=\left(0, H_{y}, 0\right)$ is directed along the $y$ axis. The components $\left(E_{x}, E_{z}, H_{y}\right)$ are determined by their Fourier decomposition in all three connected media,

$$
U_{i}(x, z, t)=\int U_{i}(x, \Omega, Q) e^{i(Q z-\Omega t)} d \Omega d Q \quad(i=1,2,3) .
$$

The Fourier amplitudes are given by

$U_{i}(x, \Omega, Q)= \begin{cases}U_{3}(\Omega, Q) e^{-\kappa_{3} x}, & x>h \\ U_{2}(\Omega, Q) e^{-\kappa_{2} x}+U_{2}^{*}(\Omega, Q) e^{\kappa_{2} x}, & 0 \leq x \leq h, \\ U_{1}(\Omega, Q) e^{\kappa_{1} x}, & x<0\end{cases}$ where

$$
\kappa_{i}^{2}=Q^{2}-\varepsilon_{i}(\Omega) \frac{\Omega^{2}}{c_{0}^{2}},
$$

with the permittivities $\varepsilon_{i}(\Omega)$ and the velocity of light in vacuum $c_{0}$. We assume translational invariance in the $y$ direction so that all field quantities are independent of the coordinate $y$.

To take into account the surface current caused by the presence of the SQDs, the boundary conditions for the waveguide modes at $x=h$,

$$
H_{y, 3}-H_{y, 2}=\frac{4 \pi}{c_{0}} \frac{\partial p}{\partial t}, \quad E_{z, 3}=E_{z, 2}
$$

and at $x=0$,

$$
H_{y, 2}=H_{y, 1}, \quad E_{z, 2}=E_{z, 1},
$$

are taken into account. The polarization $\boldsymbol{P}=\boldsymbol{e}_{z} P_{z}(x, z, t)$, $P_{z}(x, z, t)=p(z, t) \delta(x-h)$, points along the direction of the dipole moments of the quantum dots.

Supposing that the permittivities in media 1 and 3 are the same, i.e. that $\varepsilon_{1}=\varepsilon_{3}$, and using equations (1)-(5), we obtain the following nonlinear wave equation for the $E_{z}$ component of the strength of the electric field at $x=h$ :

$$
\int f(\Omega, Q) E(\Omega, Q) e^{i(Q z-\Omega t)} d \Omega d Q=-4 \pi p(z, t)
$$

where

$$
\begin{gathered}
f(\Omega, Q)=q-\frac{g^{2}}{q}, \quad q=\frac{\varepsilon_{1}}{\kappa_{1}}+\frac{\varepsilon_{2}}{\kappa_{2}} \operatorname{coth}\left(\kappa_{2} h\right), \\
g=\frac{\varepsilon_{2}}{\kappa_{2}} \frac{1}{\sinh \left(\kappa_{2} h\right)},
\end{gathered}
$$

and $E(\Omega, Q)=E_{z, 3}(h, \Omega, Q)=E_{z, 2}(h, \Omega, Q)$. Equation (6) is valid for any dependence of the polarization of the SQDs $p(z, t)$ on the strength of the electric field at $x=h$. In order to determine this dependence, we have to consider the structure of the energetic levels of the SQDs and the nonlinear interaction of the waveguide pulses with the SQDs.

The energetic structure of the SQDs is given by the ground state $|1\rangle\left(\mathcal{E}_{1}=0\right)$ and the states $|2\rangle$ and $|3\rangle$ with energies $\mathcal{E}_{2}=\hbar \omega_{0}=\mathcal{E}_{x}+\delta_{x} / 2$ and $\mathcal{E}_{3}=\hbar \Omega_{0}=2 \mathcal{E}_{x}+\delta_{x x}$, where the quantities $\mathcal{E}_{x}=\left(\mathcal{E}_{2}+\mathcal{E}_{2}^{\prime}\right) / 2$ and $\mathcal{E}_{3}$ describe the energies of the single-excitonic and biexcitonic states, respectively (see Fig. 1). $\delta_{x}=\mathcal{E}_{2}-\mathcal{E}_{2}^{\prime}$ denotes the energy of the exciton fine structure splitting and $\delta_{x x}$ the biexciton binding energy (negative if bound); $\hbar$ is Planck's constant. In the following, a parameter range is addressed where the soliton pulse spectrum is broad in comparison with both the exciton fine structure splitting (typically a few hundred $\mu \mathrm{eV}[17,18]$ ) and the biexciton binding energy (typically a few meV $[18,19]$ ) and thus the detuning between the excitonic and biexcitonic resonances. Then, both the excitonic and the biexcitonic resonances interact similarly to the pulse, and we can apply the approximation of vanishing exciton-biexciton detuning with respect to a spectrally broad pulse. Thus, we have simultaneous 


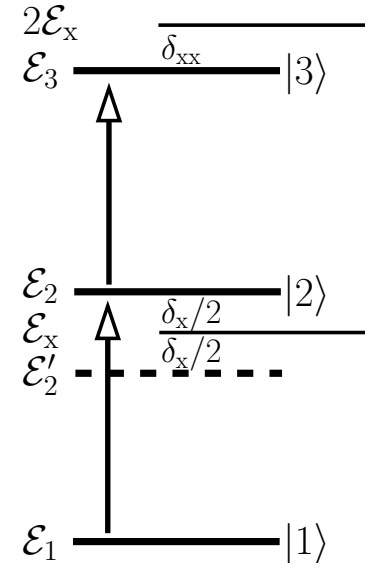

Fig. 1. Schematic sketch of the energetic structure of the semiconductor quantum dots.

resonance conditions for both the transitions $1-2$ and $2-3$. The energy spectrum of the SQDs can then be considered as a quasi-equidistant three-level system in a cascade configuration.

The system is described by the Hamiltonian

$$
H=H_{0}+V \text {, }
$$

where $H_{0}=\hbar \omega_{0}|2\rangle\left\langle 2\left|+\hbar \Omega_{0}\right| 3\right\rangle\langle 3|$ is the Hamiltonian of the single-excitonic $|2\rangle$ and biexcitonic states $|3\rangle$, and $V=-\boldsymbol{P} \boldsymbol{E}$ is the Hamiltonian of the light-quantum dot interaction. The vector of polarization is determined by interband transitions occurring in the SQDs between the three energetic levels:

$$
p(z, t)=n_{0} \int g(\Delta)\left(\mu_{12} \rho_{21}+\mu_{23} \rho_{32}\right)(\Delta, z, t) d \Delta+c . c .,
$$

where $n_{0}$ is the uniform quantum dot density, $\boldsymbol{E}=\boldsymbol{e} E$, and $\mu_{12}=\boldsymbol{\mu}_{12} \boldsymbol{e}, \mu_{23}=\boldsymbol{\mu}_{23} \boldsymbol{e}$. The dipole moments for the corresponding transitions $\boldsymbol{\mu}_{12}$ and $\boldsymbol{\mu}_{23}$ are assumed to be parallel to each other and directed along the $z$ axis. $g(\Delta)$ is the inhomogeneous broadening lineshape function resulting from the dot size fluctuations. The quantities $\rho_{i j}$ are the matrix elements of the density matrix $\rho$ determined by the Liouville equation

$$
i \hbar \frac{\partial \rho_{n m}}{\partial t}=\sum_{l}\left(\langle n|H| l\rangle \rho_{l m}-\rho_{n l}\langle l|H| m\rangle\right),
$$

where $n, m, l=1,2,3$. The resulting equations can be simplified using the method of slowly changing profiles. For this purpose, we represent the functions $E$ and $\rho_{n m}$ in the form

$$
\begin{gathered}
E=\sum_{l= \pm 1} \hat{E}_{l} Z_{l}, \\
\rho_{21}=\hat{\rho}_{21} Z_{1}, \quad \rho_{32}=\hat{\rho}_{32} Z_{1}, \quad \rho_{31}=\hat{\rho}_{31} Z_{2},
\end{gathered}
$$

where $\hat{E}_{l}$ and $\hat{\rho}_{n m}$ are the slowly varying complex amplitudes of the optical electric field and the elements of the density matrix, respectively; $Z_{l}=e^{i l(k z-\omega t)}$ with the frequency and wave number of the carrier wave $\omega$ and $k$, respectively. To guarantee that $E$ is a real number, we set $\hat{E}_{l}=\hat{E}_{-l}^{*}$. We note that such a representation of the solutions of nonlinear wave equations for pulses in semiconductors has been widely used in the theory of nonlinear waves $[9,14,20-23]$. Furthermore, in the absence of phase modulation, $\hat{E}_{l}=\hat{E}_{-l}=\hat{E}_{l}^{*} \equiv \hat{E}$. This yields the following system of equations for the slowly varying amplitudes $\hat{\rho}_{n m}$ :

$$
\begin{aligned}
& i \hbar \frac{\partial \rho_{11}}{\partial t}=\left(-\mu_{12} \hat{\rho}_{21}+\mu_{12} \hat{\rho}_{21}^{*}\right) \hat{E} \\
& i \hbar \frac{\partial \rho_{22}}{\partial t}=\left(\mu_{12} \hat{\rho}_{21}-\mu_{23} \hat{\rho}_{32}-\mu_{12} \hat{\rho}_{21}^{*}+\mu_{23} \hat{\rho}_{32}^{*}\right) \hat{E} \\
& i \hbar \frac{\partial \rho_{33}}{\partial t}=\left(\mu_{23} \hat{\rho}_{32}-\mu_{23} \hat{\rho}_{32}^{*}\right) \hat{E} \\
& i \hbar \frac{\partial \hat{\rho}_{21}}{\partial t}=\hbar\left(\omega_{0}-\omega\right) \hat{\rho}_{21}-\mu_{12} \hat{E}\left(\rho_{11}-\rho_{22}\right)-\mu_{23} \hat{E} \hat{\rho}_{31} \\
& i \hbar \frac{\partial \hat{\rho}_{32}}{\partial t}=\hbar\left(\Omega_{0}-\omega_{0}-\omega\right) \hat{\rho}_{32}-\mu_{23} \hat{E}\left(\rho_{22}-\rho_{33}\right)+\mu_{12} \hat{E} \hat{\rho}_{31} \\
& i \hbar \frac{\partial \hat{\rho}_{31}}{\partial t}=\hbar\left(\Omega_{0}-2 \omega\right) \hat{\rho}_{31}+\left(\mu_{12} \hat{\rho}_{32}-\mu_{23} \hat{\rho}_{21}\right) \hat{E} .
\end{aligned}
$$

In the above equations, the rotating wave approximation has been applied [20], and it is assumed that the SIT pulses are linearly polarized so that we can take $\mu_{12}$ and $\mu_{23}$ as real.

Since the detunings in the ensemble of different quantum dots are assumed to be equal for both transitions, $\Omega_{0}-\omega_{0}-\omega=\omega_{0}-\omega=\Delta$, where $g(\Delta)$ describes the inhomogeneous broadening, and assuming $\mu_{12}=\mu_{23}$, describing a three-level system with equal transition probabilities, equations (9) are simplified considerably to

$$
i \frac{\partial r}{\partial t}=\Delta r+\dot{\Theta} N, \quad i \frac{\partial N}{\partial t}=\dot{\Theta}\left(r-r^{*}\right),
$$

where

$$
r=\hat{\rho}_{21}+\hat{\rho}_{32}, \quad N=\rho_{33}-\rho_{11},
$$

and $\Theta(z, t)=\frac{\mu_{12}}{\hbar} \int_{-\infty}^{t} \hat{E}\left(z, t^{\prime}\right) d t^{\prime}$ is the area of the pulse. The imaginary part of the quantity $r$ is typically factorized as $\operatorname{Im} r(\Delta, z, t)=F(\Delta) \operatorname{Im} r(0, z, t)[9,20,21,23]$, where $F(\Delta)$ is the dipole spectral response function which, as in the case of the two-level system, is Lorentzian $[20,24,25]$.

In order to solve the wave equation (6), we perform a series expansion of the function $f(\Omega, Q)$ (Eq. (7)) at the position of the carrier frequency and wave number:

$f(\Omega, Q)=f(\omega, k)+(\Omega-\omega) f_{\Omega}^{\prime}(\omega, k)+(Q-k) f_{Q}^{\prime}(\omega, k)+\ldots$,

with

$$
f_{\Omega}^{\prime}=\left.\frac{\partial f}{\partial \Omega}\right|_{\Omega=\omega, Q=k}, \quad f_{Q}^{\prime}=\left.\frac{\partial f}{\partial Q}\right|_{\Omega=\omega, Q=k} .
$$

Substituting the expansion (10) in the wave equation (6) and dividing the real and imaginary parts of the equation, we obtain, taking into account that for waveguide modes $\kappa_{2}=i\left|\kappa_{2}\right|$ (cf. Eq. (3)), the dispersion law for the waveguide mode,

$$
f(\omega, k)=0,
$$

and the nonlinear wave equation

$$
\frac{\partial \hat{E}}{\partial t}+v_{g} \frac{\partial \hat{E}}{\partial z}=-\frac{4 \pi n_{0} \mu_{12}}{f_{\Omega}^{\prime}} \int g(\Delta) F(\Delta) \operatorname{Im} r(0, z, t) d \Delta .
$$


Here,

$$
v_{g}=-\frac{f_{Q}^{\prime}}{f_{\Omega}^{\prime}}
$$

is the group velocity of the linear waveguide mode. The dispersion law (12) coincides with the dispersion law for linear waveguide modes [26,27].

We can transform the wave equation (13) to the form

$$
\left(\frac{d \hat{E}}{d \zeta}\right)^{2}=T^{-2} \hat{E}^{2}-\frac{\mu_{12}^{2}}{2 \hbar^{2}} \hat{E}^{4}
$$

with $\zeta=t-\frac{z}{V}$ and the constant pulse velocity $V$. The width of the pulse $T$ is determined by

$$
T^{-2}=\frac{4 \pi n_{0} \mu_{12}^{2}}{f_{\Omega}^{\prime}\left(\frac{v_{g}}{V}-1\right) \hbar} \int \frac{g\left(\Delta^{\prime}\right) d \Delta^{\prime}}{1+\Delta^{2} T^{2}}+\mathcal{O}\left(\Delta^{2}\right) .
$$

The solution of (15) for the envelope function has the form $[9,15]$

$$
\hat{E}=\frac{2}{\mu_{0} T} \operatorname{sech} \frac{t-\frac{z}{V}}{T},
$$

where $\mu_{0}=\sqrt{2} \frac{\mu_{12}}{\hbar}$. From equation (16), we can determine the delay of the $2 \pi$ pulse on a unit length in the resonance medium:

$$
\frac{1}{V}-\frac{1}{v_{g}}=\frac{4 \pi n_{0} \mu_{12}^{2}}{\hbar f_{\Omega}^{\prime} v_{g}} \int \frac{g\left(\Delta^{\prime}\right) d \Delta^{\prime}}{T^{-2}+\Delta^{\prime 2}}+\mathcal{O}\left(\Delta^{2}\right) .
$$

Equations $(1-3,12,14,17,18)$ determine the parameters of the waveguide soliton for any value of $x, z$, and $t$. For the existence of the soliton, it is necessary that the conditions $f_{\Omega}^{\prime}>0$ and $V<v_{g}$ are fulfilled.

From equations (14) and (18), we can see that the group velocity $v_{g}$ and, for a given width $T$ of the pulse, the soliton pulse velocity $V$ depend, unlike the plane wave soliton, not only on the parameters of the connected media $\varepsilon_{1}$ and $\varepsilon_{2}$, but also on the transverse structure of the waveguide mode through the function $f_{\Omega}^{\prime}$. This circumstance leads to the fact that, unlike the soliton of the plane wave, the waveguide soliton is a two-dimensional object, and the quantity $\hat{E}$ which characterizes the soliton depends on the two space coordinates $(x, z)$ and the time $t$ as well as on the transverse structure of the waveguide mode (through $f_{\Omega}^{\prime}$ ).

Using typical parameters for the pulse, the materials, and the $\mathrm{SQDs}^{1}$, a spatiotemporally resolved plot of the $z$ component of the electric field for the two-dimensional waveguide soliton is shown in Figure 2 for a fixed value of the $z$ coordinate. In addition to the envelope of the soliton, one observes the transverse structure of the $\mathrm{TM}_{0}$ mode, antisymmetric about the center of the waveguide. Most of the energy (amplitude) of the soliton is concentrated inside of the waveguide and exponentially decreases

1 Parameters for the numerical simulation: $\omega=10^{15} \mathrm{~Hz}, T=$ $1.5 \times 10^{-13} \mathrm{~s}, h=4 \times 10^{-5} \mathrm{~cm}, \mu_{12}=2 \times 10^{-17} \mathrm{esu} \mathrm{cm}, n_{0}=$ $8 \times 10^{15} \mathrm{~cm}^{-3}, n_{1}=3.15, n_{2}=3.3$, full-width half-maximum inhomogeneous broadening $\hbar \delta^{*}=60 \mathrm{meV}$.

\section{E [a.u.]}

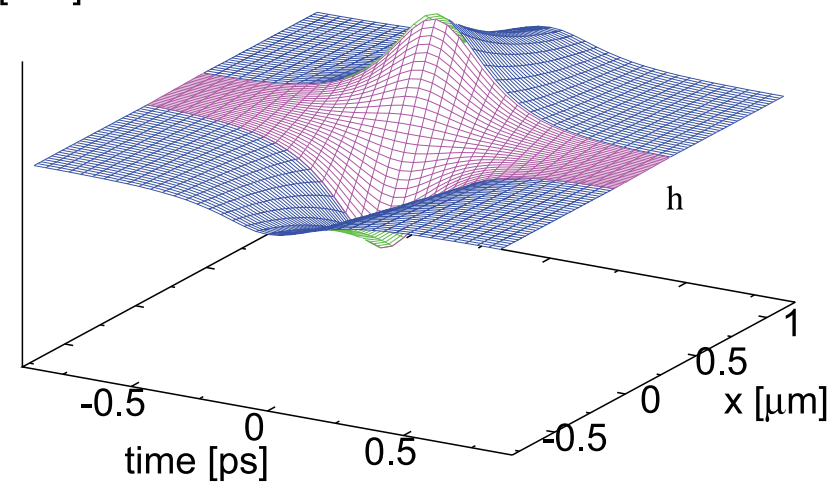

Fig. 2. (Color online) Plot of the $z$ component of the electric field at a fixed value of the $z$ coordinate showing the envelope of the soliton and the transverse structure of the TM mode.

when moving away from the surface. For the parameters used above, we find a soliton velocity of $V \approx 10^{9} \mathrm{~cm} / \mathrm{s}$ and a peak intensity of several $\mathrm{GW} / \mathrm{cm}^{2}$. Compared to the waveguide breather solution of reference [15], the soliton velocity $V$ and pulse width $T$ are not independent variables.

In conclusion, we have predicted the existence of optical waveguide solitons in a three-level SQD system for experimentally relevant parameters. We hope that the presented results will stimulate the research of twodimensional solitons in quantum dot nanostructures due to their importance for the lossless and shape-invariant transport of information on nanoscales. It should be noted that the constructed theory is quite general and can be simplified for atomic systems, for example, for dielectrics with optically active three-level impurities, in which the dipole moments are directed along the border division between the media.

We thank the Deutsche Forschungsgemeinschaft for financial support through the Sfb 787 and the DFG-Projekt 435 GEO $113 / 10 / 0-1$ as well as the ISTC.

\section{References}

1. M.A. Nielsen, I.L. Chuang, Quantum Computation and Quantum Information (Cambridge University Press, Cambridge, 2000)

2. D. Bimberg, M. Grundmann, N.N. Ledentsov, Quantum Dot Heterostructures (Wiley, Chichester, 1999)

3. P. Borri, W. Langbein, S. Schneider, U. Woggon, R.L. Sellin, D. Ouyang, D. Bimberg, Phys. Rev. B 66, 081306 (2002)

4. B. Krummheuer, V.M. Axt, T. Kuhn, Phys. Rev. B 65, 195313 (2002)

5. J. Förstner, K.J. Ahn, J. Danckwerts, M. Schaarschmidt, I. Waldmüller, C. Weber, A. Knorr, Phys. Stat. Sol. B 234, $155(2002)$

6. J. Förstner, C. Weber, J. Danckwerts, A. Knorr, Phys. Rev. Lett. 91, 127401 (2003) 
7. A. Krügel, V.M. Axt, T. Kuhn, P. Machnikowski, A. Vagov, Appl. Phys. B 81, 897 (2005)

8. S. Schneider, P. Borri, W. Langbein, U. Woggon, J. Förstner, A. Knorr, R.L. Sellin, D. Ouyang, D. Bimberg, Appl. Phys. Lett. 83, 3668 (2003)

9. S.L. McCall, E.L. Hahn, Phys. Rev. 183, 457 (1969)

10. V.M. Agranovich, V.Y. Chernyak, V.I. Rupasov, Opt. Commun. 37, 363 (1981)

11. H. Giessen, A. Knorr, S. Haas, S.W. Koch, S. Linden, J. Kuhl, M. Hetterich, M. Grün, C. Klingshirn, Phys. Rev. Lett. 81, 4260 (1998)

12. G. Panzarini, U. Hohenester, E. Molinari, Phys. Rev. B 65, $165322(2002)$

13. A. Knorr, S. Hughes, T. Stroucken, S.W. Koch, Chem. Phys. 210, 27 (1996)

14. G. Adamashvili, A. Knorr, Opt. Lett. 31, 74 (2006)

15. G.T. Adamshvili, C. Weber, A. Knorr, N.T. Adamshvili, Phys. Rev. A 75, 063808 (2007)

16. G.T. Adamashvili, A. Knorr, Phys. Lett. A 367, 220 (2007)
17. R. Seguin, A. Schliwa, S. Rodt, K. Pötschke, U.W. Pohl, D. Bimberg, Phys. Rev. Lett. 95, 257402 (2005)

18. W. Langbein, P. Borri, U. Woggon, V. Stavarache, D. Reuter, A.D. Wieck, Phys. Rev. B 69, 101301 (2004)

19. U.W. Pohl, R. Seguin, S. Rodt, A. Schliwa, K. Pötschke, D. Bimberg, Physica E 35, 285 (2006)

20. L. Allen, J.H. Eberly, Optical resonance and two-level atoms (Dover, New York, 1987)

21. I.A. Poluektov, Y.M. Popov, V.S. Roitberg, Usp. Fiz. Nauk. 114, 97 (1974)

22. Edited by N. Bloembergen, Nelineinaia spectroskopia (Mir, Moscow, 1979)

23. A.I. Maimistov, A.M. Basharov, S.O. Elyutin, Y.M. Sklyarov, Phys. Rep. 191, 1 (1990)

24. G.L. Lamb Jr., Rev. Mod. Phys. 43, 99 (1971)

25. G.L. Lamb Jr., Elements of Soliton Theory (Wiley, New York, 1980)

26. A. Yariv, P. Yeh, Optical waves in crystals (Wiley, New York, 1984)

27. A. Yariv, Quantum Electronics (Wiley, New York, 1989) 